\section{Evaluation of the contribution of the renal capsule and cortex to kidney autofluorescence intensity under ultraviolet excitation}

\author{
Rajesh N. Raman, ${ }^{a, b, *}$ Christopher D. Pivetti, ${ }^{c}$ \\ Alexander M. Rubenchik, d Dennis L. Matthews, ${ }^{a, b, d}$ \\ Christoph Troppmann, ${ }^{c}$ and Stavros G. Demos ${ }^{b, d, e}$ \\ ${ }^{a}$ University of California, Davis, Department of Applied \\ Science, Davis, California 95616 \\ ${ }^{\mathrm{b}}$ NSF Center for Biophotonics, Sacramento, California \\ 95817 \\ 'University of California, Davis Medical Center, Department \\ of Surgery, Sacramento, California 95817 \\ ${ }^{\mathrm{d}}$ Lawrence-Livermore National Laboratory, Livermore, \\ California 94550 \\ eUniversity of California, Davis Medical Center, Department \\ of Urology, Sacramento, California 95817
}

\begin{abstract}
The use of reduced nicotinamide adenine dinucleotide $(\mathrm{NADH})$ fluorescence to gain metabolic information on kidneys in response to an alteration in oxygen availability has previously been experimentally demonstrated, but signal quantification has not, to date, been addressed. In this work the relative contribution to rat kidney autofluorescence of the capsule versus cortex under ultraviolet excitation is determined from experimental results obtained using autofluorescence microscopy and a suitable mathematical model. The results allow for a quantitative assessment of the relative contribution of the signal originating in the metabolically active cortex as a function of capsule thickness for different wavelengths.

() 2009 Society of Photo-Optical Instrumentation Engineers. [DOI: $10.1117 / 1.3094948]$
\end{abstract}

Keywords: lasers; fluorescence; tissues; microscopy; ultraviolet.

Paper 08286LR received Aug. 22, 2008; revised manuscript received Dec. 11, 2008; accepted for publication Jan. 23, 2009; published online Mar. 10, 2009.

In the cell electron transport chain, reduced nicotinamide adenine dinucleotide (NADH) is an electron carrier molecule and oxygen is the ultimate acceptor. Absence of $\mathrm{O}_{2}$ induces a shift in the NADH redox state, which is accompanied by a change in the NADH autofluorescence (AF) intensity, since its oxidized form does not fluoresce. This sensitivity gives rise to a diagnostic potential in various clinical conditions.

Measurements of the change in NADH emission under UV excitation have been documented over the last 50 years in various organs including kidney, typically as a relative change of the signal intensity following an insult. The majority of these studies have used a small animal model such as rat, ${ }^{1-3}$ in which the capsule layer surrounding the kidney tissue is thin and its contribution to the total signal may be limited. How-

*Address all correspondence to: E-mail: topraman@ucdavis.edu ever, the capsule of a human kidney can be up to several times thicker, and its contribution to the overall signal intensity through attenuation of the excitation light reaching the metabolically active cortex, as well as generation of its own AF signal, may need to be taken into account.

Despite the attention given to NADH fluorimetry, including a small number of cases on human kidney AF during transplantation, ${ }^{4}$ there has been no measurement of what proportion of the generated AF actually arises from the metabolically active kidney cortex tissue versus the capsule, and how it is affected by the capsule's thickness. Such information would enable the quantitative correlation of the change in the observed AF intensity to changes in the NADH concentration in the cells that would yield a more accurate description of the organ's metabolism and function. Signal quantification will enable the exploration of the full potential of this technology as an in vivo diagnostic tool to noninvasively obtain metabolic information in various clinical conditions, such as during surgical procedures associated with ischemic injury and organ response to reperfusion following traumatic injury or transplantation.

The objective of this work is to quantify the relative contribution in the measured AF from the cortex tissue compared to that from the capsule layer in the kidney under UV excitation using 355 and $266 \mathrm{~nm}$ as representative excitation wavelengths. Experimental results were obtained using a rat model, and a mathematical model was used to estimate the corresponding values in a human kidney.

Rat kidneys were excised and then kept in isotonic saline at $4{ }^{\circ} \mathrm{C}$ for $48 \mathrm{~h}$ to allow an equilibrium in the NADH concentration to be reached after removal (due to change in metabolism). ${ }^{5,6}$ Measurements were successfully carried out on seven kidneys total obtained from Wistar-Furth (two kidneys, two rats), Sprague-Dawley (three kidneys, two rats), and Lewis (two kidneys, one rat) rats. Images of intact kidney, kidney with capsule removed, and one and two capsule layers without kidney, were acquired. Kidney with no capsule layer was prepared by cutting the capsule along one edge and pulling back to expose the cortex. A double capsule layer was formed by folding a single capsule layer back onto itself.

Two diode-pumped UV lasers were used for photoexcitation of the kidney tissue operating at 355 and $266 \mathrm{~nm}$ delivering a dosage to the tissue of 0.10 and $0.20 \mathrm{~mJ} / \mathrm{mm}^{2}$, respectively. Exposure time for each source was $5 \mathrm{sec}$ concurrent with image acquisition. AF microscopic images were acquired using a microscopy system described in detail elsewhere. ${ }^{7}$ In brief, a liquid nitrogen-cooled camera was used to record the images that were formed using a $20 \times$ objective (Mitutoyo, Japan) followed by a $5 \times$ zoom lens after passing through a 420-nm long-pass filter. Each AF image was normalized to the fluorescence image of a quartz slide to account for variation in beam intensity over illumination spot. Intensities were averaged over three to four tubule regions near the center of the image.

Figure 1 shows typical AF images under 266- and 355-nm excitation of a rat kidney with its surface in three different configurations. Specifically, the images in Figs. 1(a) and 1(c) contain intact kidney on the top half of the image and exposed cortex on the bottom half under 266- and 355-nm excitations,

1083-3668/2009/14(2)/020505/3/\$25.00 @ 2009 SPIE 


\section{JBO LETTERS}
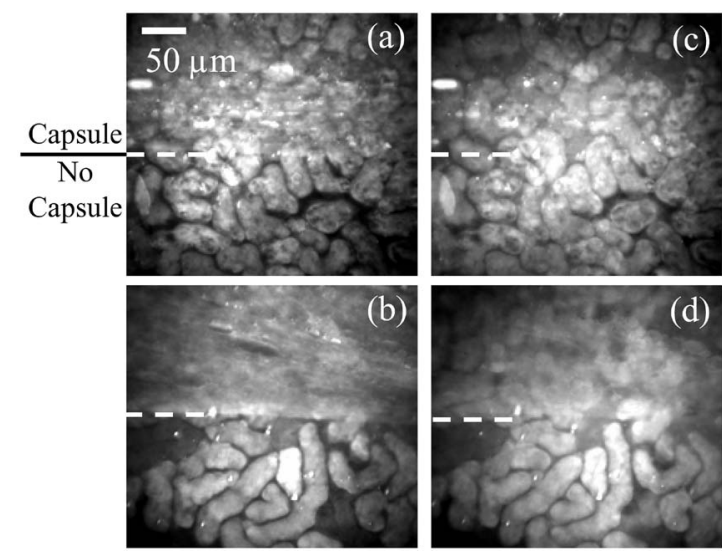

Fig. 1 Autofluorescence microscopy images of ex vivo rat kidney with capsule removed from lower half of each image. Images (a) and (b) are under 266-nm excitation, while images (c) and (d) are under 355-nm excitation. Images (b) and (d) contain an additional layer of capsule on the upper half of the kidney.

respectively. Images shown in Figs. 1(b) and 1(d) contain an additional layer of capsule on the upper half (folded single capsule layer). Features of the tubules are visible in the bottom half of each image. Under 266-nm excitation, the morphology of the tubules is partially visible beneath one layer of capsule [Fig. 1(a)], but much less so under two capsule layers [Fig. 1(b)]. The tubule morphology is visible in the corresponding images under $355 \mathrm{~nm}$, even through two capsule layers. The AF intensities for the different capsule configurations, normalized to the intensity from one capsule and averaged over the seven kidneys, are shown in Table 1.

The capsule thickness $a$ was estimated from the image shown in Fig. 1(a) (and corresponding images from all other kidneys) to be $a=25 \pm 5 \mu \mathrm{m}$ for all seven kidneys by measuring the distance in the axial direction needed to translate the kidney to move the image focus from the top of the capsule to the top of the cortex. Due to difficulty in straightening out the entire capsule layer(s), each measured intensity is an average over a region of interest of about $25 \times 25 \mu \mathrm{m}$ to avoid folds. Factors contributing to the observed standard deviations in the experimental results may be attributed in part

Table 1 Autofluorescence intensities (mean \pm SD for $n=7$ rat kidneys) normalized to that of one capsule layer. Values for $\mu_{C}\left(\mathrm{~cm}^{-1}\right)$ and $B$ were calculated from these intensities according to the model described in the text.

\begin{tabular}{lll} 
& $266 \mathrm{~nm}$ & $355 \mathrm{~nm}$ \\
1 capsule layer only & 1.00 & 1.00 \\
2 capsule layers only & $1.64 \pm 0.20$ & $1.86 \pm 0.14$ \\
1 capsule layer on kidney & $3.63 \pm 1.24$ & $7.30 \pm 2.91$ \\
2 capsule layers on kidney & $3.03 \pm 0.90$ & $6.81 \pm 2.09$ \\
$\mu_{C}\left(\mathrm{~cm}^{-1}\right)$ & $326 \pm 154$ & $116 \pm 54$ \\
$B$ & $0.300 \pm 0.133$ & $0.168 \pm 0.082$ \\
\hline
\end{tabular}

to air gaps after capsule manipulation, and in part to the presence of capsule heterogeneities such as small fatty or connective tissue residues that may have clung to the capsule.

A reasonable description of light propagation in turbid media can be obtained using simplified models. ${ }^{8}$ The light intensity decreases exponentially $\sim \exp (-\mu x)$ with tissue depth $x$, where the attenuation coefficient $\mu$ is given by the combination of the absorption length $\mu_{a}^{-1}$, scattering length $\mu_{s}^{-1}$, and scattering anisotropy $g: \mu=\mu_{a}+(1-g) \mu_{s}$. Since attenuation occurs for the incident light $I_{0}$ as well as for the AF emission, the effective attenuation coefficient is the sum of the coefficients for incident and AF light. Disregarding reflections at the tissue-air boundary, we can estimate the AF signal $F$ from kidney containing capsule of thickness $a$ over the cortex tissue, as

$$
\begin{aligned}
F & =Q_{C} \int_{0}^{a} I_{0} \exp \left(-\mu_{C} x\right) d x+Q_{T} \int_{0}^{\infty} I_{0}(a) \exp \left(-\mu_{T} x\right) d x \\
& =\frac{Q_{C}}{\mu_{C}} I_{0}\left[1-\exp \left(-\mu_{C} a\right)\right]+\frac{Q_{T}}{\mu_{T}} I_{0} \exp \left(-\mu_{C} a\right),
\end{aligned}
$$

where $Q$ is a constant describing the emission efficiency per unit area of the tissue, and indices $C$ and $T$ refer to capsule and tubules in cortex, respectively. The proportion of the signal from the cortex is given by

$$
\frac{F_{T}}{F_{C}+F_{T}}=\left[1+\frac{B}{\mu_{C} a} \times \frac{1-\exp \left(-\mu_{C} a\right)}{\exp \left(-\mu_{C} a\right)}\right]^{-1},
$$

where the dimensionless constant $B=\left(Q_{C} \mu_{T} a\right) / Q_{T} . B$ (which is proportional to the ratio of $\mathrm{AF}$ conversion efficiency of capsule to that of cortex) and $\mu_{C}$ correspond to optical properties of the capsule and cortex and are independent of illumination and collection geometry.

By folding the isolated capsule and additionally making a measurement of the AF signal with double capsule thickness $2 a$, the capsule attenuation coefficient $\mu_{C}$ and the parameter $B$ can be measured from Eq. (2) for each incident wavelength by using a mean value of $a=25 \mu \mathrm{m}$ and the mean intensity values from the images (Table 1). These constants were then used to plot the estimated dependence of the contribution of the cortex AF to the total signal [Eq. (2)] for arbitrary capsule thickness in Fig. 2, where the value for contribution of the tubules at $0-\mu \mathrm{m}$ capsule thickness was defined as $100 \%$. Values at 25 and $50 \mu \mathrm{m}$ were obtained from the intensities from one and two capsule layers, respectively.

Based on direct measurements ${ }^{9,10}$ and histological results, ${ }^{11}$ we assumed the average human kidney capsule thickness to be on the order of $100 \mu \mathrm{m}$. In addition, the human kidney capsule has a similar composition and structure to that of the rat kidney capsule comprised of dense connective tissue primarily of collagenous fibrils embedded with elastin fibers. ${ }^{12}$ Therefore, we postulate that the results obtained from rat kidneys can be used to estimate the corresponding AF signal intensities in human kidneys. A previous study of the attenuation coefficient of collagenous tissue reported values from 50 to $500 \mathrm{~cm}^{-1}$ over an excitation range of 248 to $532 \mathrm{~nm},{ }^{13}$ consistent with our results. The estimated percent contribution of the cortex to the total detected AF intensity as a function of capsule thickness under 266- and 355-nm excitations (aver- 


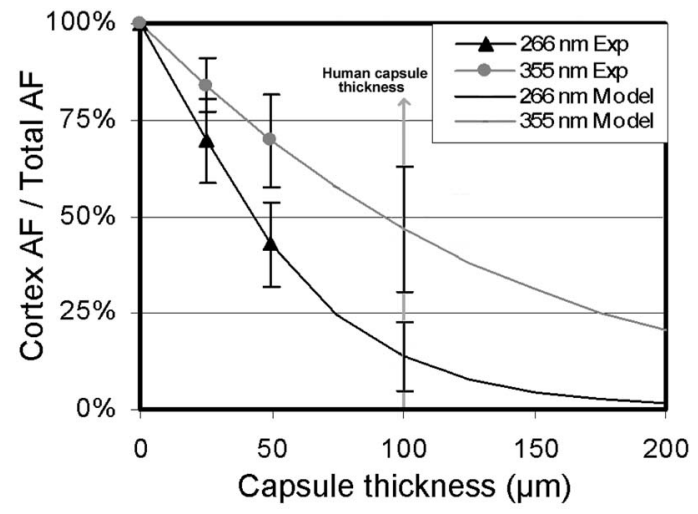

Fig. 2 Plots of the percent contribution of the cortex to the total detected autofluorescence signal versus capsule thickness (mean values, $n=7$ ) under 266- and 355-nm excitations. Arrow indicates estimated values for human kidney. Standard deviation bars are indicated at 25-, $50-$, and $100-\mu \mathrm{m}$ capsule thicknesses. $A F=$ autofluorescence intensity, and $\operatorname{Exp}=$ experimental.

aged over the seven kidneys) is shown in Fig. 2. Standard deviation at 25 and $50 \mu \mathrm{m}$ are based on mean values from the measurements. Standard deviations for values at thicknesses $>50 \mu \mathrm{m}$ are based on mean values from the modeling results. The values at $100 \mu \mathrm{m}$ represent the estimated values for a human kidney.

The results suggest that about half of the detected AF in a human kidney under 355-nm excitation would arise from the cortex. This finding also enables signal quantification to correlate measured changes in signal intensity to changes in cellular NADH concentration. For signal quantification, it is also necessary to account for changes induced by variable illumination-collection geometry and changes in the absorption by the blood (arising from changes in the oxygenation level or blood volume). There have been several methods proposed to address this issue. One approach is to subtract the scattered excitation light signal from the fluorescence signal. ${ }^{2}$ This method can be accurate only when the probe is in contact with the tissue, but can give rise to additional signal artifacts, such as localized variations from the presence of microvasculature. Another approach is to monitor reflectance near hemoglobin isosbestic wavelengths in the near-IR region. ${ }^{1}$ However, this method probes deeper into the kidney than the UV excitation and may monitor blood volume changes occurring beneath the kidney cortex where hemodynamic properties are known to differ.

Using a non-contact optical probe, the amount and angular distribution of the reflected light contains an unknown mixture of diffuse and specular components that can change with illumination-collection geometry and hydration state, potentially creating signal artifacts independent of tissue metabolism when the organ is exposed during clinical procedures. Since AF under 266-nm excitation would primarily originate in the human capsule ( $\sim 88 \%$ of signal) and arise mainly from biomolecules not involved in metabolism, its measured intensity would depend on probe-tissue geometry and specular reflection changes corresponding to variable hydration state. ${ }^{14}$ Additionally, the capsule would effectively screen the 266-nm light, which in sufficient doses can be harmful to cortex tissue. ${ }^{15}$ Blood absorption would have to be estimated by another method, such as analyzing the strength of blood absorption features in the broad NADH emission spectrum.

In conclusion, the results presented in this work address the issue of signal quantification of NADH AF as a tool to noninvasively obtain metabolism and function-related information from kidneys. In vivo studies will help refine and validate the methods discussed in this work.

\section{Acknowledgments}

This work was performed under the auspices of the U.S. Department of Energy by Lawrence Livermore National Laboratory under contract DE-AC52-07NA27344 and the Center for Biophotonics, an NSF Science and Technology Center managed by the University of California, Davis, under Cooperative Agreement Number PHY 0120999. The authors wish to thank the laboratory of Sarah Yuan of the Division of Research, Department of Surgery, UC Davis School of Medicine, for assistance with tissue samples.

\section{References}

1. S. Kobayashi, K. Kaede, K. Nishiki, and E. Ogata, "Microfluorometry of oxidation-reduction state the rat kidney in situ," J. Appl. Physiol. 31(5), 693-696 (1971).

2. A. Mayevsky and B. Chance, "Intracellular oxidation-reduction state measured in situ by a multichannel fiber-optic surface fluorometer," Science 217(4559), 537-540 (1982).

3. J. M. C. C. Coremans, M. Van Aken, D. C. W. H. Naus, M. F. Van Velthuysen, H. A. Bruining, and G. J. Puppels, "Pretransplantation assessment of renal viability with NADH fluorimetry," Kidney Int. 57(2), 671-683 (2000).

4. A. Mayevsky, J. Sonn, M. Luger-Hamer, and R. Nakache, "Real-time assessment of organ vitality during the transplantation procedure," Transplant Rev. (Philadelphia) 17(2), 96-116 (2003).

5. G. M. Palmer, C. L. Marshek, K. M. Vrotsos, and N. Ramanujam, "Optimal methods for fluorescence and diffuse reflectance measurements of tissue biopsy samples," Lasers Surg. Med. 30(3), 191-200 (2002).

6. K. T. Schomacker, J. K. Frisoli, C. C. Compton, T. J. Flotte, J. M. Richter, N. S. Nishioka, and T. F. Deutsch, "Ultraviolet laser-induced fluorescence of colonic tissue: basic biology and diagnostic potential," Lasers Surg. Med. 12(1), 63-78 (1992).

7. S. G. Demos, C. A. Lieber, B. Lin, and R. Ramsamooj, "Imaging of tissue microstructure using a multimodal microscope design," IEEE J. Sel. Top. Quantum Electron. 11(4), 752-758 (2005).

8. S. Jacques, "Simple optical theory for light dosimetry during PDT," Proc. SPIE 1645, 155-165 (1992).

9. J. G. Snedeker, P. Niederer, F. R. Schmidlin, M. Farshad, C. K. Demetropoulos, J. B. Lee, and K. H. Yang, "Strain-rate dependent material properties of the porcine and human kidney capsule," J. Biomech. 38(5), 1011-1021 (2005).

10. H. Yamada, Strength of Biological Materials, G. Evans, Ed., Williams and Wilkins, Huntingdon, NY (1970).

11. Cell and Tissue Biology: A Textbook of Histology, 6th ed., L. Weiss, Ed., Urban and Schwarzenberg, Baltimore, MD (1988).

12. R. E. Bulger, "Rat renal capsule: presence of layers of unique squamous cells," Anat. Rec. 177(3), 393-403 (1973).

13. F. W. Cross, R. K. Al-Dhahir, and P. E. Dyer, "Ablative and acoustic response of pulsed UV laser-irradiated vascular tissue in a liquid environment," J. Appl. Phys. 64(4), 2194-2201 (1988).

14. J. T. Fitzgerald, A. P. Michalopoulou, C. D. Pivetti, R. N. Raman, C. Troppmann, and S. G. Demos, "Real-time assessment of in vivo renal ischemia using laser autofluorescence imaging," J. Biomed. Opt. 10(4), 044018 (2005).

15. Y. Matsumura and H. N. Ananthaswamy, "Short-term and long-term cellular and molecular events following UV irradiation of skin: implications for molecular medicine," Expert Rev. Mol. Med. 4, 1-22 (2002). 